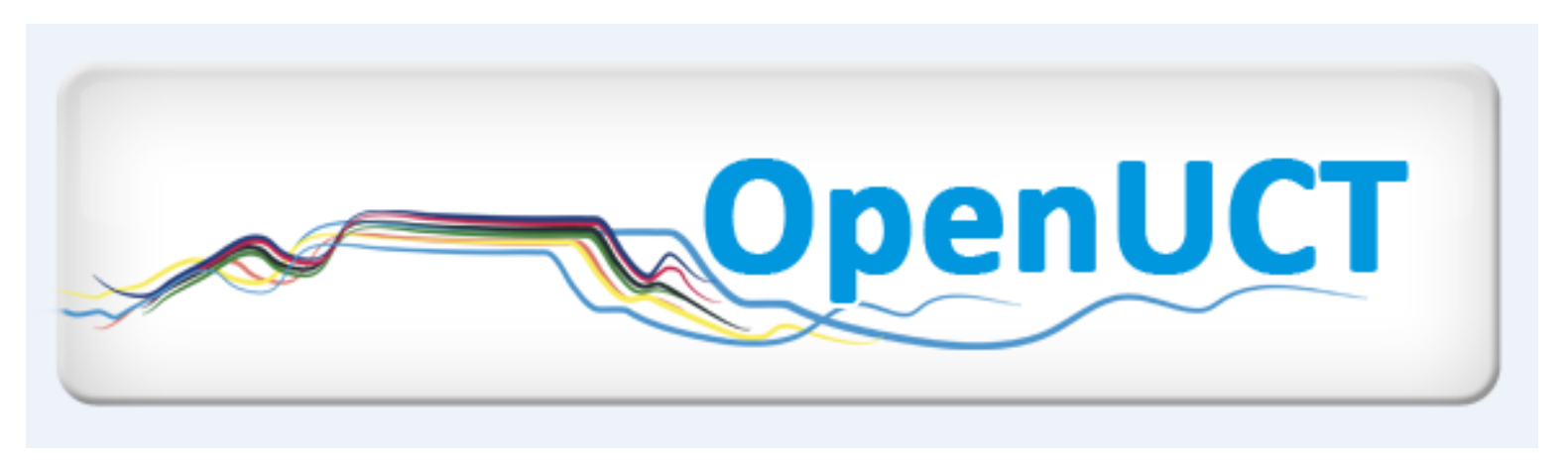

This is the author-approved manuscript version of a journal article published in:

Bohlmann, C. A. \& Fletcher, L. 2008. Diagnostic assessment for mathematics in a distance learning context. South African Journal of Higher Education. 22(3): 556-574. DOI: 10.4314/sajhe.v22i3.25802.

It is made available under the terms of agreement between the author and the journal, and in accordance with the University of Cape Town's Open Access Policy for the purposes of research, teaching and private study. http://www.openuct.uct.ac.za/sites/default/files/UCTOpenAccessPolicy.pdf 


\title{
Diagnostic assessment for mathematics in a distance learning context
}

\author{
Carol A. Bohlmann \\ Centre for Higher Education, Department of Mathematical Sciences, \\ University of Cape Town, South Africa \\ e-mail: carol.bohlman@uct.ac.za
}

Lizelle Fletcher

Department of Statistics, University of Pretoria, South Africa.

e-mail: liezel.fletcher@up.ac.za

\begin{abstract}
The idea of a diagnostic process to identify students likely to benefit from additional support originated from two separate investigations relating to the performance of entry-level mathematics students at the University of South Africa (UNISA). One project explored the relationship between reading skill and mathematical performance. This resulted in a reading intervention programme for students of mathematics. The other project considered an alternative method of assessment. This article explains why and how diagnostic assessment was developed for students who enrolled for the UNISA Mathematics Access Module. The article gives some initial the results, and an analysis of examination performance related to the risk categories identified by the diagnostic assessment.
\end{abstract}

\section{INTRODUCTION}

Historically there has been a disparity in the availability of educational opportunities for South African students, resulting in the unreliability of matriculation results as predictors of academic performance. The predicament of "disadvantaged" learners has received greater attention in South Africa since 1994, when, for the first time, race was not a barrier to entering an educational institution. The term "disadvantaged" ' is normally used 'to describe students whose schooling has been negatively affected by (mis)education or other circumstances such as poor socio-economic and political conditions. Problems often manifest themselves in communication ..., cognitive ..., and subject-specific deficiencies' (Mphalhele 1994, 49). It follows that not all applicants in tertiary education meet the required criteria, or are likely to be successful. 
Over the years various alternative admission routes have thus been explored to accommodate students who do not meet formal entry requirements (Foxcroft 1999). The government also recognises that institutions 'will continue to have the right to determine entry requirements as appropriate beyond the statutory minimum. However ... selection criteria should be sensitive to the educational backgrounds of potential students ...' (Government Gazette 18 April 1997, No. 17944).

As a distance education institution, UNISA offers different routes into mainstream mathematics for students who do not meet set entry criteria. A bridging mathematics module, which took students from Grade 12 Standard Grade (SG) mathematics to Higher Grade (HG) mathematics and slightly beyond, was introduced in 1993. A mathematics access module 2 was introduced in 1997. This module takes students from Grade 9 mathematics to Grade 12 SG mathematics and includes some HG mathematics topics but not trigonometry or Euclidean geometry. 3 See figure 1.

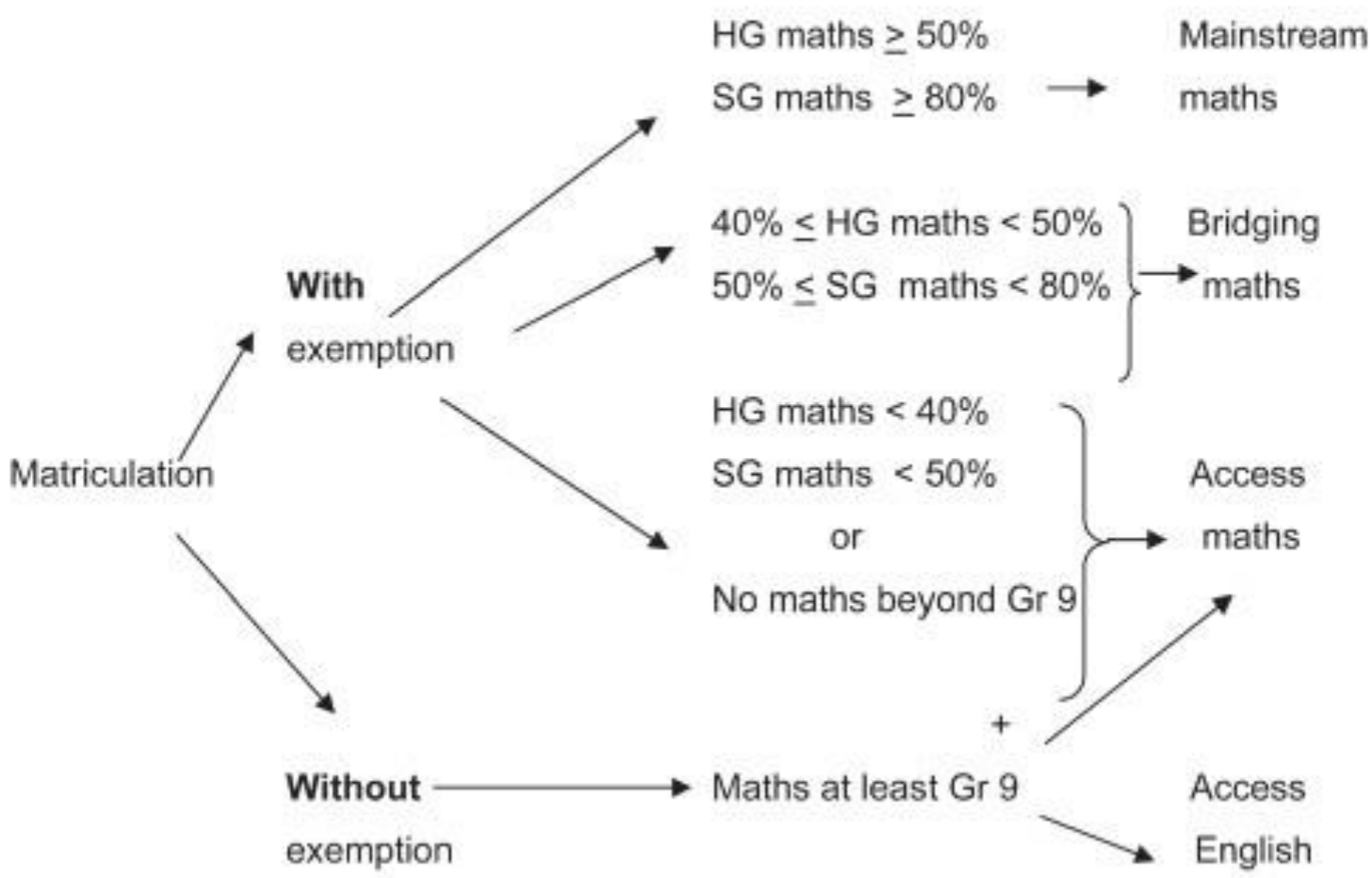

Figure 1: Entry into mathematics at UNISA 5

The figure shows that students could begin studying the Mathematics Access Module with limited mathematical background knowledge. In many cases they were from disadvantaged backgrounds, with the additional hurdles that this implies. Over the years pass rates were low and attrition rates high. This led to a joint project involving the Departments of Mathematical Sciences and Linguistics in which reading skill was investigated as a possible cause for poor performance (Bohlmann and Pretorius 2002). Two reading interventions were implemented. The findings of a face-to-face intervention programme (Bohlmann and Pretorius 2003) highlighted the 
need of some form of diagnostic assessment to identify students who could benefit from additional support. This was further supported by the results of an alternative assessment project (Bohlmann 2006).

\section{BACKGROUND}

\section{Reading skill and mathematics}

A reading intervention programme with three distinct phases was implemented for Mathematics Access Module students during the period 2000-2003. Phase I (2000) involved initial investigations with a small group of volunteers, the development of a reading test and analyses of the test results after it was administered to all students registered for the module (Bohlmann and Pretorius 2002). The most important result from Phase I was that students' reading proficiency and examination performance were directly related, that is, the stronger a student's reading ability, the better his/her mathematics examination performance. While high reading scores do not guarantee mathematical success, a low reading score seemed to be a barrier to success.

Phase II (2001) saw the development of a face-to-face intervention programme, involving volunteer students (Bohlmann and Pretorius 2003). Aspects such as anaphoric referencing, logical relations, academic vocabulary and visually presented information were addressed. The Phase II pretests showed that the students entered the reading programme with low reading ability. They were reading at an average speed of 92 words per minute, well below the acknowledged recommended speed of 160 words per minute. They also had poor comprehension levels (average 46\%). It seemed that students had not been sufficiently exposed to print, and did very little reading beyond what was strictly necessary for study purposes. After the 22-week intervention period, the post-tests showed a 10 per cent increase in comprehension levels. However, with a mean score of 56 per cent, the majority of students would still have been regarded as weak readers. An analysis of the relationship between the students' reading skills and examination marks confirmed the findings from Phase I, where it had been established that students with reading scores below 60 per cent would probably fail.

Phase II suggested that the intervention should be more intensive (i.e. more hours a week) and longer (e.g. for a full year); it also needed to be more relevant to the distance learning context. The third phase (Phase III, 2002-2003) thus involved the production of a video and a supplementary video workbook, which focused specifically on the reading skills needed to make sense of mathematics discourse. However, feedback after the first two attempts at using the video and workbook (Bohlmann 2003) suggested that this intervention had limited impact, as many students did not, or could not, use the material effectively on their own. 


\section{General knowledge and meta-cognition}

A parallel analysis (2001-2003) of the results of an alternative form of assessment for Mathematics Access Module students highlighted a number of meta-cognitive and general knowledge problems which also appeared to be a barrier to mathematical achievement (Bohlmann 2006). Without an adequate frame of reference students found it difficult to make sense of real-life examples used to illustrate mathematical concepts; furthermore they often did not recognise when their arguments contained contradictions or were unintelligible.

\section{A RATIONALE FOR DIAGNOSTIC ASSESSMENT}

Entry-level assessment can provide information that will facilitate smoother articulation from school to tertiary study by identifying students who are unlikely to study mathematics successfully without additional support. The results noted above supported the idea that a diagnostic process was required to identify such students. It was thought that pre-registration assessment could raise prospective students' awareness regarding their levels of academic and quantitative literacy (i.e. familiarity with and facility in the use of academic discourse, and in the use and interpretation of numerical information). It could enable the institution to provide better guidance regarding appropriate support, and motivate students to take advantage of such support. The results noted above (see the section 'General knowledge and meta-cognition' above) also suggested several aspects that could be investigated in a diagnostic assessment process.

The main goal of diagnostic assessment is that students should be accurately placed in categories according to potential risk. In the United Kingdom, Appelby and Cox (2002) suggest that effective teaching requires that lecturers know at what level their students are when they enter a course of study. This leads to the idea of a survey of incoming students, 'to audit their strengths and weaknesses' (Appelby and Cox 2002, 12). The information obtained can be used to channel potentially 'at risk' students into support programmes with regard to basic numerical, reading and academic skills before studying mathematics. So-called 'risk-free' students could probably cope with the support inherent in the study programme. Assessment for appropriate placement would also serve to alleviate the perception that such an assessment might serve as a gate-keeping mechanism.

Testing in various forms takes place at many institutions, nationally and internationally, in the form of aptitude and interest tests, proficiency tests, diagnostic tests, etcetera. In the United States of America (USA) Scholastic Achievement Tests (SATs) are regularly used (Isaacs 2001); Israeli universities use a psychometric test as an additional admissions requirement (Beller 2001). In Sweden admission to higher education for students under the age of 25 is based on school performance or the results of an entrance examination (Baumslag 2000).

There are several South African examples of entry-level testing. The tests used in the Alternative Admissions Research Project at the University of Cape Town (UCT) 
were first introduced in a pilot project in 1987, and have subsequently been expanded to one of the currently most effective and widely used means of assessment (Cliff, Visser, Hanslo and Yeld 2003). A battery of placement tests has been effectively used at the Nelson Mandela Metropolitan University (NMMU) since 1999 (Foxcroft 2004). In the College of Science at the University of the Witwatersrand, which accommodates educationally disadvantaged students, selection tests led to the first two years of four-, five-, or seven-year degrees (NARSET Report 1997). A placement test specifically for mathematics students was introduced at the University of the Western Cape (UWC) 'positively as a means of inclusion rather than exclusion' (Amoah 1998, 47). The validity of this test was investigated over the three-year period from 1994 to 1996, and it was concluded that there was a positive relationship between the placement test and student performance in one of the firstyear mainstream mathematics courses.

\section{ACCEPTANCE OF DIAGNOSTIC ASSESSMENT AT UNISA}

In the light of declining pass rates and increasing attrition rates despite increasing support, the UNISA Senate approved the introduction of diagnostic assessment (Mathematics Diagnostic Assessment (MDA)) for all potential Mathematics Access Module students in June 2003. Once this decision had been taken, it was necessary to consider what aspects the MDA would include. Based on local and international practices, and the UNISA results noted in Section 2 above, reading skill and appropriate numerical competence appeared to be the most important aspects.

In spite of concerns that a satisfactory assessment process could not be established in the limited time available (between Senate approval and the finalisation of all necessary procedures) it was clear that commencement in 2004 was justified since it could assist students, and would provide useful information regarding procedures, processes and structures that were practical within the UNISA context.

The MDA was not approved as a prerequisite to study, but simply as a coregistration requirement. Students who registered too late to write the MDA at the beginning of the year were required to write mid year, resulting in two groups.

Students in the mid-year group were thus assessed after exposure to some teaching. The two groups were incorporated as two strata in the statistical analyses.

\section{INVESTIGATING THE VARIOUS OPTIONS}

Developing diagnostic tests is costly and time-consuming. Therefore it was decided to investigate some of the existing tests mentioned in Section 3, as these were already based on internationally accepted standards and had been screened for reliability and validity.

Two tests were identified that could be adapted to suit specific UNISA requirements, viz. the UCT Alternative Admissions Research Project (AARP) tests, and the battery of placement tests used at the NMMU. 


\section{The Alternative Admissions Research Project, UCT}

UCT began the process of designing, refining and using AARP tests in the late 1980s. These tests stretch over most of one day, and consist of a 'Placement Test in English for Educational Purposes' (PTEEP), a 'Mathematics Comprehension Test' (MCom) and a "Mathematics Achievement Test" (MAch). The PTEEP and the MCom assess potential rather than knowledge, whereas the MAch relies heavily on subject content knowledge (Cliff, Visser, Hanslo and Yeld 2003). In these tests, results are analysed, and adjustments are made according to a set formula to smooth out differences in educational background.

The MAch was at too advanced a level for potential UNISA Mathematics Access Module students. In a trial undertaken at UNISA in August 1998 the PTEEP and MCom appeared to have predictive value for success in the Mathematics Access Module (Yeld, private communication).

It was necessary for Unisa to offer diagnostic assessment at all of its examination centres. In a distance-learning context, computerised testing is possibly the most efficient and effective means of carrying out diagnostic assessment. Even though UNISA was not yet in a position to provide computerised testing for all potential students, this option was investigated further

Student circumstances and UNISA's registration practices presented a number of potential problems. Many first-time students tended to register as late as possible: students waited for matriculation results and/or finance, and only register just before the closing date (end of February or early in March). This has implications for any pre-registration assessment process, in that sufficient time is needed to coordinate all the activities related to writing and processing the assessment, and disseminating the results.

The logistics involved in administering several different examinations over an extended registration period, coordinating delivery of scripts by UCT to UNISA's examination centres, and collecting and returning the scripts to UCT for processing, would be enormous. Furthermore, the AARP tests are not electronically available. Therefore, even though they may have been the most appropriate in the UNISA context, practical considerations precluded their implementation at UNISA.

\section{Testing for placement at the NMMU}

The NMMU was established in 1969 as a bilingual (English and Afrikaans) university. In 1997 its multilingual student population comprised a diverse mix of students from different population groups (39\% Black, 16\% Asian and Coloured and $45 \%$ White); by 1999 the percentage of Black students had increased to 52 per cent (Foxcroft 1999). The NMMU draws most of its students from the Eastern Cape, a relatively economically impoverished region (GCIS 2003). At entry level many of its students thus have similar characteristics to UNISA students (such as variable levels of schooling, diversity of language and culture, registration several years after leaving school). 
The NMMU used a battery of placement tests, piloted in 1999. Several of the NMMU test criteria were equally applicable to the UNISA context: a test battery should take into account cultural, language and educational background; recognise potential, rather than acquired knowledge and skills; meet standard psychometric criteria (i.e. provide credible measurements of student cognitive competence); be researched on an ongoing basis to establish predictive validity, reliability and suitability, and be checked for bias (Foxcroft 1999).

At NMMU the competencies that appeared to be most closely related to academic success were academic literacy, English language proficiency, numeracy, and certain non-cognitive factors such as motivation and post-school experience (Foxcroft 1999). The NMMU used ACCUPLACER Computerized Placement Tests, obtained from the Educational Training Service in the USA, to assess academic literacy, language proficiency and mathematical skill. The ACCUPLACER language assessment has two components: an English Language Proficiency Test and a Reading Comprehension Test. Mathematical skills were assessed using the Basic Arithmetic Test and the Basic Algebra Test. The ACCUPLACER algebra, arithmetic and reading tests are computerised adaptive tests, i.e. item response theory is used to generate a unique test for each student. Written versions (called the COMPANION) are available for students unfamiliar with computers. The paper and pencil version only has one set of questions. The NMMU has used both types, and has carried out research into the different test formats.

Based on the ACCUPLACER test results, a risk profile is established for each student, on the basis of which academic performance can be predicted, developmental needs can be established and early remedial action can be taken where necessary (Koch and Foxcroft 2002).

ACCUPLACER tests can be obtained via the Internet from the College Board's agent and can also be purchased in booklet form. The option of computerised delivery and marking of the ACCUPLACER tests could be seen as a significant potential advantage in the long term, unless local tests become widely available. A significant disadvantage would be the cost (US dollar related) of purchasing the tests (either the printed tests, or the 'right' to be tested, if the online version was selected). However, the opportunity of providing students with appropriate advice and facilitating throughput should be weighed against attrition and failure.

\section{COMPONENTS OF THE ASSESSMENT}

As explained above, it was decided that diagnostic assessment at UNISA would focus on reading skill and quantitative reasoning ability.

\section{Selection of ACCUPLACER for reading comprehension}

The NMMU experience led to the decision to use the ACCUPLACER Reading Comprehension (ARC) Test. In Phase II of the UNISA reading intervention it had been demonstrated that reading effectively was fundamental to constructing meaning from 
mathematics texts. When students have difficulty reading to learn, it is often assumed that their limited language proficiency is the primary cause. This reflects an underlying assumption that language proficiency and reading ability are equivalent, which is not the case. Research has shown that improving language proficiency does not necessarily improve reading (Hacquebord 1994). Language proficiency and reading are related; however, they are conceptually and cognitively uniquely specific skills that develop in distinct ways. It thus seemed that the ACCUPLACER English Language Proficiency Test was possibly less useful for the MDA purposes than the ARC test.

At NMMU the reading tests were used in their American format, but an empirical item bias investigation was initiated to identify items that were possibly in need of adaptation. Initially NMMU developed their own standards on the tests, and did not use the USA norms or standards. At UNISA the standards used in interpreting ARC scores were also changed slightly, as explained in the next section.

Table 1 shows the different aspects of reading assessed in the ARC Test. Clearly, several questions tested more than one aspect; furthermore there is some overlap between categories.

Table 1: Aspects of reading tested in the ARC Test

\begin{tabular}{|l|c|}
\hline Aspect tested & Number of occurrences \\
\hline Causal relations & 2 \\
\hline Contrastive relations & 1 \\
\hline Recognition of sequence & 9 \\
\hline Interpretation of implied or inferred information & 5 \\
\hline $\begin{array}{l}\text { Comprehension/interpretation of factual information; } \\
\text { detailed/general. }\end{array}$ & 12 \\
\hline Identification of main/subsidiary ideas & Many (in 33 questions) \\
\hline Academic vocabulary & 1 \\
\hline Number sense & 2 \\
\hline Recognition of contradiction, inconsistency & 1 \\
\hline Substantiation & \\
\hline
\end{tabular}

Although anaphoric resolution had been identified as an important skill (Bohlmann and Pretorius 2002; Bohlmann and Pretorius 2003), this was not directly assessed in the ARC Test. Since accurate anaphoric resolution is integral to reading comprehension it was perhaps not essential to assess this aspect directly; however further investigation into these and other results could lead to their inclusion of specific anaphoric items later. 
A number of questions included potentially ambiguous words or phrasing, or cultural bias. However, academics in the Departments of Linguistics and Practical English agreed that this would not necessarily detract from the potential of the items to assess students' ability to construct meaning. As these tests are used internationally, and validity and reliability are assured, it was decided to leave the items in their original format for the first round of testing, and delete or adapt them if the test results suggested that this was necessary.

\section{Selection of a quantitative reasoning test}

The level of content knowledge in the Mathematics Access Module was considerably lower than that reflected by the mathematical sections of many other placement or diagnostic tests, including the ACCUPLACER Basic Arithmetic Test and the Basic Algebra Test. The attraction of the AARP MCom was that it does not draw on previously taught mathematics. It is a dynamic test that primarily assesses reasoning potential, and it includes limited mathematical knowledge that could realistically be expected from students. However, the AARP tests were not an option for UNISA, and the ACCUPLACER tests were unsuitable. It was thus necessary to design a UNISA-specific quantitative literacy test, the Basic Arithmetic Test (BAT), which needed to comprise questions which emphasised understanding and reasoning above recall, similar to the AARP MCom.

The BAT questions were hence structured to determine whether sufficient understanding of basic concepts had been achieved, as students who had not understood the rudimentary concepts taught up to the end of Grade 9 would have had little chance of understanding the mathematics content of the Mathematics Access Module.

Some questions were designed to test common misunderstandings that had been identified as significant barriers to understanding the module content. Questions were also based on the results of a research project undertaken by a lecturer in the Department of Statistics at UNISA involving students enrolled in a first-year mathematics module and their ability to improve their mathematics performance through drill-and-practice mathematics instruction (Rossouw 2006).

Questions involving time appeared in the Mathematics Access Module study material. Several questions were thus included in the test to probe students' understanding of time (with respect to "when" and to "how long"). For example, 'Joseph paints a cupboard in $2 \frac{1}{2}$ hours. Sydney paints twice as fast as Joseph. How long does it take Sydney to paint the cupboard?'. When the reading speed of Mathematics Access Module students was assessed in 2002 and 2003, students were asked to note their starting and finishing times, and hence calculate the total time (the difference these two). A number of students added the two times. This gave rise to the following BAT question: 'Maria starts to read a magazine article at 8:30. She finishes the article at 10:00. How long does it take her to read the article?'. 
The ability to recognise pattern is another important aspect in comprehending mathematical concepts (Freeman 1994), and this was also assessed. Academic literacy within the subject domain is important. Students must, for example, be able to make mathematical sense out of phrases such as "three times as heavy as", "at least", "greater than'. Several questions featured phrases such as these, such as the paint example above.

The BAT questions varied in difficulty from simple computational tasks to activities requiring insight, reasoning skill, and the ability to interpret simple sentences mathematically. The question categories are shown in Table 2. As in the ARC Test, several questions tested more than one aspect, with some overlap between categories.

Table 2: Aspects of quantitative reasoning in the Basic Arithmetic Test (BAT)

\begin{tabular}{|l|c|}
\hline Aspect tested & Number of occurrences \\
\hline Simple arithmetic operations (whole numbers) & 19 \\
\hline Simple arithmetic operations (fractions/decimals) & 3 \\
\hline Pattern recognition & 5 \\
\hline Number sense & 3 \\
\hline Conversion of units & 3 \\
\hline $\begin{array}{l}\text { Academic vocabulary in relation to mathematics (e.g. } \\
\text { satisfy) }\end{array}$ & 4 \\
\hline Technical vocabulary (e.g. zero, fraction, ratio) & 10 \\
\hline $\begin{array}{l}\text { Comparison (e.g. bigger than, earlier, more than half, } \\
\text { double) }\end{array}$ & 3 \\
\hline Time (how long) & 2 \\
\hline Time (when) & 17 \\
\hline 'Translation' from words to mathematical statements & 4 \\
\hline Recognition of insufficient or redundant information & 2 \\
\hline Learning from explanation & 4 \\
\hline Spatial awareness & \\
\hline Insight & \\
\hline
\end{tabular}

Obviously reliability and validity could not be ensured in the same way as for the AARP and ACCUPLACER tests, however questions were carefully selected and discussed within a wide group of academics. 


\section{CATEGORISATION}

Criteria needed to be set to categorise students who were considered likely to be successful in the Mathematics Access Module with no additional assistance (Low Risk), those likely to be successful provided they had additional support (Medium Risk) and those unlikely to be successful without assistance beyond that available at the university (High Risk). The NMMU had developed a profile across all scores in the battery (including school performance) and used regression formulae and classification functions to classify potential students as being high, medium or low risk. These formulae were developed on the basis of previous research (Koch, Foxcroft and Watson 2001; Koch and Foxcroft 2002). At UNISA the criteria for classification were based on the ACCUPLACER guidelines, and empirical results following Phases I, II and III of the reading intervention (Bohlmann and Pretorius 2002; Bohlmann and Pretorius 2003; Bohlmann 2003).

\section{Criteria for the Reading Comprehension Test}

The ACCUPLACER Coordinator's Guide provided a table to convert the raw score out of 35 to a score out of 120 (Administrator's Manual 2002). Some form of weighting clearly takes place. Students with between no and four correct answers out of 35 obtain a score of 20 points. The increment between the numbers of correct answers increases gradually from 1 point to 4 points. Students are thus rewarded for obtaining a greater number of correct answers.

The ACCUPLACER recommendations provide three categories: the weakest reflects a score of about 21 or less out of 120; about 80 out of 120 is regarded as moderate, and about 103 or higher is good. These scores reflect increasing levels of reading skill, outlined in the ACCUPLACER Coordinator's Guide.

Scoring 80 on a scale from 20 to 120 is roughly 60 per cent. Phases I and II of the reading intervention suggested that a score of 60 per cent appeared to be a threshold below which reading skills were too weak to support effective study. In the MDA the high-risk cut-off point (students at risk with respect to reading) was pitched slightly lower, at 70 out of 120 (58\%), equivalent to a raw score of 23 out of 35.

\section{Criteria for the Basic Arithmetic Test}

Questions were weighted, with the simplest, procedural questions (such as addition or multiplication) being given weights of 1 or 1,5 and questions requiring some insight and interpretation of language being given weights of $2,2,5$ or 3 , respectively. Students who demonstrated greater insight could thus score better than those who had merely learnt to memorise procedures.

When the raw score out of 35 was converted according to these weights the final maximum score was 69 . Table 3 shows the distribution of questions with different weights and the contribution of the different categories to the total. 
Table 3: Weight distribution of questions in Basic Arithmetic Test

\begin{tabular}{|l|c|c|c|l|}
\hline Category & Weight & $\begin{array}{c}\text { Percentage } \\
\text { of total }\end{array}$ & $\begin{array}{c}\text { Number of } \\
\text { items }\end{array}$ & Aspect assessed \\
\hline Easy & 1 & 10 & 7 & $\begin{array}{l}\text { Simple arithmetic } \\
\text { (whole numbers) }\end{array}$ \\
\hline Easy & 1,5 & 13 & 6 & $\begin{array}{l}\text { Simple arithmetic with fractions/ } \\
\text { decimals }\end{array}$ \\
\hline $\begin{array}{l}\text { Moderately } \\
\text { easy }\end{array}$ & 2 & 29 & 10 & $\begin{array}{l}\text { Arithmetic, number sense, } \\
\text { language, time }\end{array}$ \\
\hline $\begin{array}{l}\text { Moderately } \\
\text { difficult }\end{array}$ & 2,5 & 22 & 6 & $\begin{array}{l}\text { Arithmetic, number sense, } \\
\text { language, time, money, insight }\end{array}$ \\
\hline Difficult & 3 & 26 & 6 & $\begin{array}{l}\text { Arithmetic, number sense, } \\
\text { language, time, money, pattern, } \\
\text { insight }\end{array}$ \\
\hline
\end{tabular}

From this table it can be seen that students could score up to 52 per cent by correctly answering easy to moderately easy items (with weights of 1, 1,5 or 2), and up to 74 per cent by correctly answering easy, moderately easy and moderately difficult items (with weights up to 2,5). Students who scored over 74 per cent also needed to answer some of the difficult items (with weights of 3) correctly.

Ten items (with weights accounting for $17 \%$ of the total) were purely computational and required no reading skills. It should have been possible for all students to answer these questions correctly. There were thus 25 items ( $83 \%$ of the total) that depended to some extent on reading, as well as other skills. Considering the 60 per cent threshold identified with respect to reading comprehension, it seemed reasonable to set 17 per cent $+(60 \%$ of $83 \%)$, that is, 67 per cent, as the lowest boundary for this component of the assessment. This is equivalent to a raw score of 46 out of 69 . Students with a score of less than 46 were thus considered as being at risk with respect to quantitative reasoning.

The top category in the ARC comprises scores of 103 (approximately 83\%) or higher. To achieve such scores in the BAT, students needed to score 57 or more out of 69 . With no other empirical evidence this was selected as the cut-off point for high achievement in the BAT.

\section{Risk categories}

The following table was used to determine risk categories. 
Table 4: Diagnostic assessment categories

\begin{tabular}{|l|l|l|l|}
\hline & $\begin{array}{l}\text { Reading Comprehension } \\
(\text { weighted) }(20-120)\end{array}$ & $\begin{array}{l}\text { Basic Arithmetic (weighted) } \\
(0-69)\end{array}$ \\
\hline High Risk & Score $<70$ & OR & Score $<46$ \\
\hline Low Risk & Score $\geq 103$ & AND & Score $\geq 57$ \\
\hline Medium Risk & All other scores \\
\hline
\end{tabular}

\section{ADMINISTRATION OF THE MDA}

On registration students were provided with details regarding the date and venue of the assessment. They also received information regarding the purpose and the implications of the assessment, and suggestions for of support options for those placed in high or medium risk categories. There was no assessment fee. Those responsible for marketing were asked to disseminate MDA information widely and frequently. Information also appeared in printed documentation regarding UNISA registration requirements.

Ideally, a test battery should not take longer than two and a half to three hours to complete. Since the ARC Test (Part 1) contained 35 questions, it was decided that the BAT (Part 2) would also contain 35 questions, as it seemed reasonable to expect students to be able to manage 70 questions in the allocated three-hour period.

At the time of writing the MDA, each student received the ARC Test, the BAT, a mark-reading sheet, and a list of instructions. The reading items were numbered 1 to 35 and the BAT items, answered on the same mark-reading sheet, were numbered 36 to 70 . Students received both parts together and could answer questions in any order. All questions were multiple-choice, each with four options. Space was available on the question papers for rough work. Invigilators were asked to ensure that question papers were returned with the mark-reading sheets, primarily for security reasons, as the same tests were used later. For research purposes it was essential that all students wrote the same assessment.

\section{RESULTS}

Only 326 students were assessed in January 2004. Another 834 students wrote in June. Despite the fact that the MDA was compulsory, 358 of the 1518 students who registered for the Mathematics Access Module in 2004 did not write the test. The examination performance across the three risk categories of the students who wrote the assessment in January and June respectively, as well as the performance of those who were not assessed, was compared to establish whether there were any differences between the groups. 


\section{Distribution of students across the risk categories}

Distribution of students across the three risk categories is shown in Table 5.

Table 5: Distribution of students across the risk categories

\begin{tabular}{|l|c|c|c|}
\hline & Frequency (\%) & January 2004 (\%) & June 2004 (\%) \\
\hline High Risk & $846(73)$ & $223(68)$ & $623(75)$ \\
\hline Medium Risk & $269(23)$ & $93(29)$ & $176(21)$ \\
\hline Low Risk & $45(4)$ & $10(3)$ & $35(4)$ \\
\hline Total & $1160(100)$ & $326(100)$ & $834(100)$ \\
\hline
\end{tabular}

The ratio 223/326 shows that approximately 68 per cent of the students who wrote the MDA in January were allocated to the High Risk category. This high figure was not unexpected, since in the previous year 76 per cent of the Mathematics Access Module students were unsuccessful (failed or dropped out).

More surprising was the analysis of the June results, which showed that 75 per cent of these students were placed in the High Risk category. Thus, in spite of a few months of exposure to the mathematics study material (and in many cases also to study material for an equivalent English module), allocation of students to the different categories followed a similar pattern to that of the January group.

\section{Examination results}

Of the 1518 students who registered for the Mathematics Access Module, 912 were not admitted to the examination. Some of them dropped out during the year. It is reasonable to assume that the majority of these 912 students did not cancel their registration but failed to gain examination admission (based on scoring a specified minimum number of credits in assignments submitted during the year). Of the 606 who obtained examination admission, 42 did not write the examination. There were thus 564 students who wrote the examination, reflecting the alarming attrition rate within this group. Of these only 163 students passed: 144 in October 2004 and 19 in the supplementary examination in January 2005, that is, approximately 29 per cent of those who wrote the examination.

Table 6 displays the distribution of the students' pass rates across the three risk categories, as well as the distribution of students who did not write the MDA.

In total 1160 students wrote the MDA. Of these only 463 wrote the examination. In the Low Risk category, 66 per cent of the students passed the examination as opposed to 45 per cent and 18 per cent for the Medium Risk and High Risk categories respectively. The pass rate of the 101 students who did not write the MDA but wrote the examination fell between that of the High and Medium Risk students. 
Table 6: Distribution of pass/ fail over risk category

\begin{tabular}{|l|c|c|c|}
\hline & Pass (\%) & Fail (\%) & Total (\%) \\
\hline High Risk & $61(18)$ & $271(82)$ & $332(100)$ \\
\hline Medium Risk & $46(45)$ & $56(55)$ & $102(100)$ \\
\hline Low Risk & $19(66)$ & $10(34)$ & $101(100)$ \\
\hline No MDA & $37(37)$ & $64(63)$ & $564(100)$ \\
\hline Total & $163(29)$ & $401(71)$ & \\
\hline
\end{tabular}

The mean examination score of the 463 assessed students was 35 per cent, while that of the group who did not write the diagnostic assessment was 39 per cent. However, closer scrutiny of the examination results of the assessed students across the different categories showed that the students who did not write the MDA did not fare much better than the students in the High Risk category, and fared more poorly than those in the Medium Risk category. Table 7 displays the mean examination results across the three risk categories. It appears that the MDA succeeded in identifying those students who were least likely to succeed.

Table 7: Examination results

\begin{tabular}{|l|c|c|}
\hline & Frequency (\%) & Average \% \\
\hline High Risk & $332(59)$ & 45 \\
\hline Medium Risk & $102(18)$ & 56 \\
\hline Low Risk & $29(5)$ & 39 \\
\hline No MDA & $101(18)$ & 36 \\
\hline Total & $564(100)$ & \\
\hline
\end{tabular}

The median analysis concurred with the means analysis. The box plots in Figure 2 showed the poor performance of the students who were not assessed and of the students in the High Risk category, relative to the other two categories. The median scores are displayed as thick horizontal lines while outliers are shown separately on the box plots. It is interesting to note that the students who were assessed in January performed more poorly than students who were assessed in June, although the differences between the meridians were negligible for the High Risk group. 


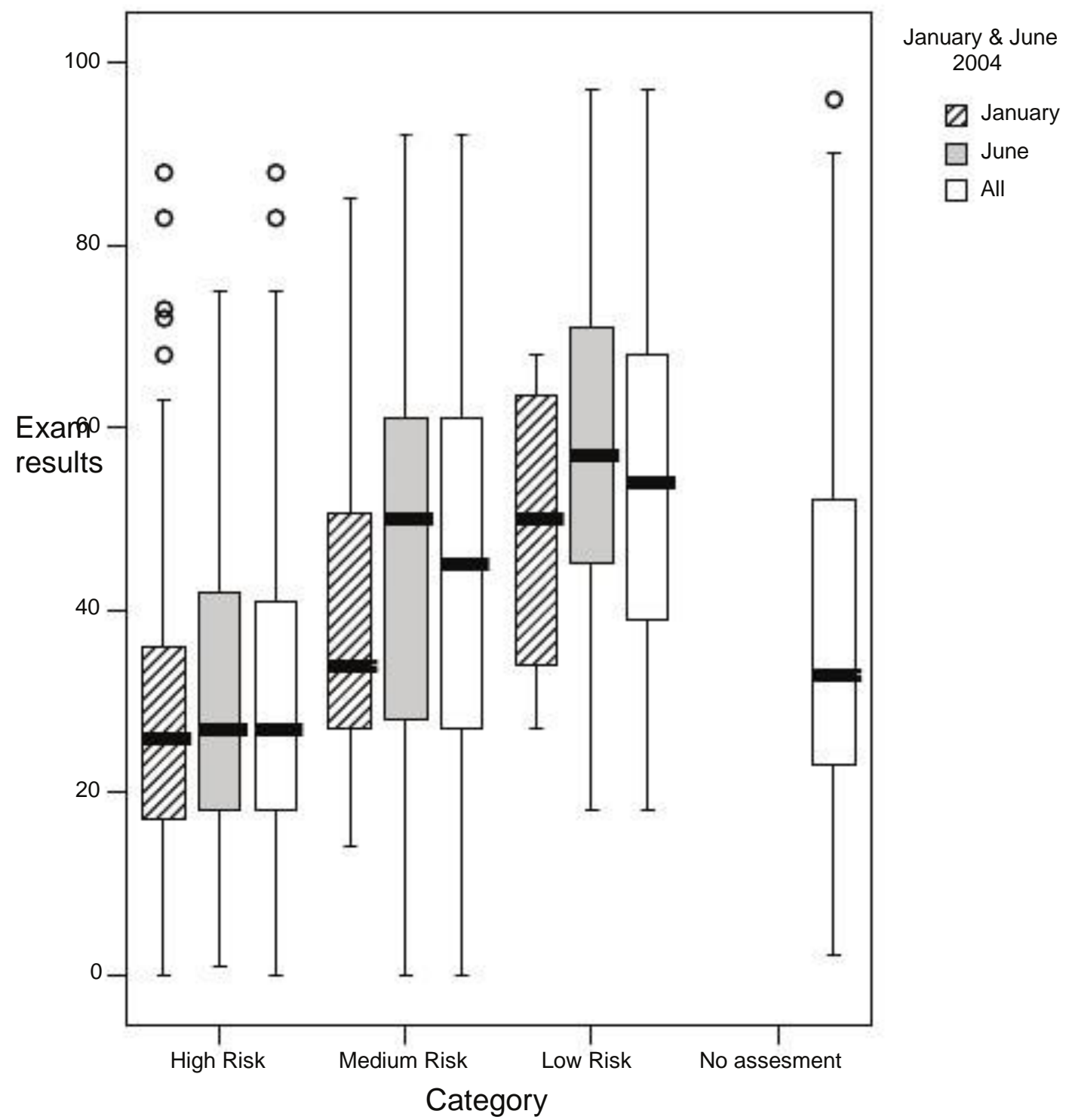

Figure 2: Box plots of examination results

\section{Distribution of MDA students who did not write the examination}

To further judge whether the categorisation process was useful, the distribution of the 698 students who did not write the examination was analysed across the different categories (see Table 8). The proportion of students without examination admission for each category is displayed as a percentage in the last column. An alarming 74 per cent of the students in the High Risk category (514 of the 846) failed to gain examination admission, thus suggesting that the MDA correctly identified the "at risk" students. This observation was confirmed by interaction with students once the examination admissions had been finalised. It emerged that many of the students in the High Risk category had been unable to obtain examination admission, in spite of submitting all assignments. 
Table 8: $\quad$ Students not admitted to the examination

\begin{tabular}{|l|l|l|l|}
\hline & $\begin{array}{l}\text { Number (\%) per } \\
\text { category }\end{array}$ & $\begin{array}{l}\text { Number (\%) } \\
\text { without admission }\end{array}$ & $\begin{array}{l}\text { Percentage per } \\
\text { category without } \\
\text { admission }\end{array}$ \\
\hline High Risk & $846(73)$ & $514(74)$ & 61 \\
\hline Medium Risk & $269(23)$ & $164(23)$ & 61 \\
\hline Low Risk & $45(4)$ & $20(3)$ & 44 \\
\hline Total & $1160(100)$ & $698(100)$ & \\
\hline
\end{tabular}

The lower percentage of students in the Low Risk category who did not obtain examination admission (20 out of 45, i.e. $44 \%$ ) stands in strong contrast to the 61 per cent of students without examination admission in the other two categories, but is nevertheless disturbingly high. It is possible that students in the High Risk category who cancelled their registrations benefited from the MDA, in that they at least did not persevere for an entire year, and yet fail. Further investigation into their circumstances may have been valuable but could not be undertaken. Students in the Medium Risk and Low Risk categories continued their studies, but did not avail themselves of support (or for whom no support was available) and did not obtain examination admission could not have benefited from the diagnostic assessment process.

Anecdotal evidence from students who were in contact with academic staff showed that those in the High Risk category on the whole least understood the purpose of diagnostic assessment and were experiencing many problems. (It cannot, however, be assumed that they represented all students in this category).

\section{CONCLUSION}

This first attempt at implementing diagnostic assessment at UNISA highlighted the complexity of this process at a distance teaching institution. The results of the categorisation process were encouraging. The distribution of the pass rate across the three risk categories (cf. Table 6) shows that the assessment succeeded to some extent in discriminating amongst the students who were likely to succeed with no additional assistance (Low Risk) and those who needed additional support (Medium Risk and High Risk). Furthermore, the Low Risk category students performed better in the examination than did the students in the other two categories (cf. Table 7), with an average score of above 50 per cent. These results support the ability of the categorisation process to separate students who need additional support in order to pass from those who can cope by themselves. Extensive statistical analyses of the examination performance and assignment results of the 2004 and possibly 2005 cohorts, such as cluster analysis, logistic regression and discriminant analysis, with a 
view to refining and improving the MDA and the categorisation process, are ongoing and would have been useful but was not possible.

All quantitative research should be supplemented by means of qualitative research such as questionnaires and focus group interviews. Such research can yield richer information regarding students' perceptions of the MDA and its effect, and suggest ways in which how assessment can become more meaningful and more widely accepted. It is hoped that this course of action can be implemented in the future.

\section{NOTES}

1. This reasearch was undertaken within the Unisa context, while the two authors were attached to the then Department of Mathematics and Statistics respectively. It describes an attempt at creating diagnostic assessment for a particular group of students at Unisa, but has implication for such assessments in contact universities as well.

This was at first an access examination, but evolved into a fully taught module.

2. The ACCUPLACER guide uses this terminology: the conversions do not make scores

3. of exactly 51,80 or 103 possible. Clearly some students would have scored less than 51 : presumably a small minority who were not functionally literate, who would probably not have been expected to take the test.

The entry requirements have changed since this research was undertaken, and will in the near future change further to accomodate learners who have taken Mathematical

4. Literacy at school.

Note that this will change once the categories HG and SG are abolished.

5.

\section{REFERENCES}

Administrators' Manual for the Companion to ACCUPLACER, The College Board. 2002.

Amoah, V. K. 1998. Prediction of first year mathematics course performance from mathematics placement test at the University of the Western Cape. In Proceedings of the Sixth Annual Meeting of the Southern African Association for Research in Mathematics and Science Education, eds. N. A. Ogude and C. Bohlmann, 47-49. University of South Africa.

Appleby. J. and W. Cox. 2002. The transition to higher education. In Effective learning and teaching in mathematics and its applications, eds. P. Kahn and J. Kyle, 3-19. The Institute for Learning and Teaching in Higher Education, The Times Higher Education Supplement. Kogan Page.

Baumslag, B. 2000. Education systems in brief. In Fundamentals of teaching Mathematics at University Level, 199-205. Imperial College Press, Sweden.

Beller, M. 2001. Admission to higher education in Israel and the role of the psychometric entrance test: Educational and political dilemmas. Assessment in Education 8 (3): 315-337.

Bohlmann, C. A. 2003. How do learners construct meaning when they read mathematics? International Journal of Learning 10:3243-3261.

- 2006. An assessment experiment for Mathematics at the University of South Africa (UNISA). South African Journal of Higher Education 20(4).

Bohlmann, C. A. and E. J. Pretorius. 2002. Reading skills and mathematics. South African Journal of Higher Education 16 (3): 196-206. 
2003. A reading intervention programme for mathematics students. South African Journal of Higher Education 17 (2): 226-236.

Cliff, A, A. Visser, M. Hanslo and N. Yeld. 2003. The balancing of excellence with equity: The Alternative Admissions Research Project. Unpublished report, University of Cape Town.

Foxcroft, C. D. 1999. Placement assessment: Lessons learned in a South African context. Paper presented at the $9_{\text {th }}$ Annual National ACCUPLACER Conference, Denver, Colorado, 29-31 July, 1999.

. C D 2004. Admissions and placement assessment programme (APAP). Unpublished report, University of Port Elizabeth (now the Nelson Mandela Metropolitan University).

Freeman, R. 1994. How to learn Maths. National Extension College, London.

GCIS, see Government Communication and Information System.

Government Communication and Information System. 2003. Pocket guide to South Africa. STE Publishers, Pretoria. Available at: http://www.gcis.gov.za.

Government Gazette. 1997. 18 April 1997, no. 17944.

Hacquebord, H. 1994. AL reading in the content areas: text comprehension in secondary education in The Netherlands. Journal of Research in Reading 17:83-98.

Isaacs, T. 2001. Entry to university in the United States: The role of SATs and advance placement in a competitive sector. Assessment in Education 8 (3): 391-406.

Koch, E., C. Foxcroft and A. Watson. 2001. A developmental focus to student access at the University of Port Elizabeth: process and preliminary insights in placement assessment. South African Journal of Higher Education 15 (2): 126-131.

Koch, S. E. and C. D. Foxcroft. 2002. A developmental focus to admissions testing: admissions and placement standards development. South African Journal of Higher Education 17 (3): 192-208.

Mphalhele, M. K. 1994. Access, equity and redress in Science Academic Development programmes: Critical issues and concerns. In Projects speak for themselves 1993/1994 (2nd edition), Science and mathematics education in the transition, ed. S. Levy, 49-54. Houghton.

NARSET, see National Access and Retention in Science, Engineering and Technology.

National Access and Retention in Science, Engineering and Technology Report. 1997. Issues relating to access and retention in science, engineering and technology in higher education. Proceedings of a consultative conference, Department of Arts, Culture, Science and Technology (DACST) and the Foundation for Research and Development (FRD), Pretoria.

Rossouw, M. H. 2006. Focused training for Mathematics Access Module students. Departmental Report 01/2006, Department of Statistics, UNISA. 